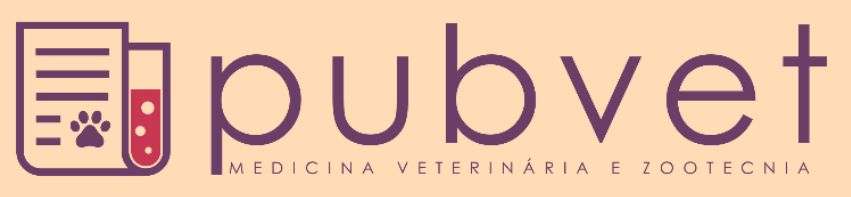

https://doi.org/10.31533/pubvet.v13n5a325.1-7

\title{
Diagnóstico de atendimento das normas de bem-estar animal em abatedouro de frangos situado no estado de Goiás
}

\author{
Paulo Vinícius da Costa Mendes ${ }^{1 *} \bullet$, Heloisa Pinto de Godoy Siqueira ${ }^{2} \bullet$, André Buzutti de \\ Siqueira $^{3}$, Luiz Francisco Prata ${ }^{4} \oplus$

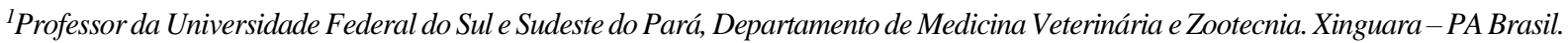 \\ ${ }^{2}$ Professora da Universidade Federal de Roraima, Departamento de Medicina Veterinária. Boa Vista - RR Brasil. \\ ${ }^{3}$ Professor da Universidade Federal de Roraima, Departamento de Medicina Veterinária. Boa Vista - RR Brasil. \\ ${ }^{4}$ Professor Adjunto II da Faculdade de Ciências Agrarias e Veterinárias da Universidade estadual paulista, Departamento de \\ Medicina Veterinária Preventiva. Jaboticabal - SP Brasil. \\ *Autor para correspondência, e-mail: paulo.vinicius@unifesspa.edu.br
}

\begin{abstract}
Resumo. Bem-estar é um termo de uso corrente em várias situações e seu significado é compreendido de modo relativo. Entretanto, definição objetiva de bem-estar faz-se necessária para a utilização científica e profissional do conceito. Bem-estar deve ser definido de forma que permita pronta relação com outros conceitos, tais como: necessidades, liberdades, felicidade, adaptação, controle, capacidade de previsão, sentimentos, sofrimento, dor, ansiedade, medo, tédio, estresse e saúde. Objetiva-se apresentar um diagnóstico de situação atinente ao atendimento dos conceitos de bem-estar desde a recepção até a sangria de frangos abatidos em abatedouro-frigorífico situado em Goiás.
\end{abstract}

Palavras-chave: bem-estar, diagnóstico, frango, recepção, sangria

\section{Diagnosis of compliance with animal welfare standards in broiler slaughterhouse located in the state of Goiás}

\begin{abstract}
Welfare is a term commonly used in various situations and its meaning is understood in relative terms. However, objective definition of well-being is necessary for the scientific and professional use of the concept. Well-being should be defined in a way that allows a ready relationship with other concepts, such as: needs, freedoms, happiness, adaptation, control, predictability, feelings, suffering, pain, anxiety, fear, boredom, stress and health. The objective of this study was to present a diagnosis of the situation regarding the welfare concepts from the reception to the bleeding of poltry slaughtered in a slaughterhouse located in Goiás.
\end{abstract}

Keywords: welfare, diagnosis, poultry, reception, bleeding

\section{Diagnóstico sobre la implementación de las normas de bienestar animal en planta de sacrificio de pollos situado en el estado de Goiás}

Resumen. El bienestar es un término de uso corriente en varias situaciones y su significado se entiende de modo relativo. Sin embargo, la definición objetiva de bienestar se hace necesaria para la utilización científica y profesional del concepto. El bienestar debe ser definido de forma que permita una pronta relación con otros conceptos, tales como: 
necesidades, libertades, felicidad, adaptación, control, capacidad de predicción, sentimientos, sufrimiento, dolor, ansiedad, miedo, aburrimiento, estrés y salud. Se pretende presentar un diagnóstico de situación relativa a la implementación de los conceptos de bienestar desde la recepción hasta la sangría de pollos faenados en la planta de sacrificio situada en Goiás.

Palabras clave: bienestar, diagnóstico, pollo, recepción, sangría

\section{Introdução}

O mercado consumidor vem passando por uma transformação significativa: atender à crescente valorização do bem-estar animal, com uma demanda de conhecimento e atuação reconhecida em várias áreas de estudo que fundamentam as profissões das ciências agrárias que trabalham desde a produção até o abate (Dawkins, 2017). Segundo o Decreto № 9.667/2019, as atribuições sobre bem-estar animal de produção e de interesse econômico são de natureza e competência da Secretaria de Defesa Agropecuária e segurança de alimentos do Ministério da Agricultura, Pecuária e Abastecimento no caso da fiscalização e normatização. O tema é considerado de importância especial por muitas pessoas; porém, requer uma definição estrita se a intenção é a sua utilização de modo efetivo e consistente (Gregory, 2003; Gregory \& Grandin, 2007). Um conceito claramente definido de bem-estar é necessário para utilização em medições científicas precisas, em documentos legais e em declarações e discussões públicas. Para que o bem-estar possa ser comparado em situações diversas ou avaliado em uma situação específica, deve ser medido de forma objetiva (Grandin, 2014). Um critério essencial para a definição de bem-estar animal útil é que a mesma deve referir-se a característica do animal individual, e não a algo proporcionado ao animal pelo homem (Grandin, 2014). O bem-estar do animal pode melhorar como resultado de algo que lhe seja fornecido, mas o que se lhe oferece não é, em si, bem-estar. Os efeitos sobre o bem-estar incluem aqueles provenientes de doença, traumatismos, fome, estimulação benéfica, interações sociais, condições de alojamento, tratamento inadequado, manejo, transporte, procedimentos laboratoriais, mutilações variadas, tratamento veterinário. O objetivo deste trabalho é apresentar os requisitos e o conceito para definição de bem-estar e, na sequência, um diagnóstico de situação, sob a égide dos atos regulatórios, de um abatedouro de aves no interior de Goiás.

\section{Materiais e métodos}

\section{Local de obtenção dos dados}

A realização deste trabalho foi possível pela anuência de um abatedouro-frigorífico de Aves e Coelhos habilitado à exportação, localizado na região sudoeste do Estado de Goiás. O estabelecimento de abate tem capacidade de abate de 470.000 aves por dia, com velocidade média de 9.000 aves por hora, divididas em três linhas de abate.

Dentre as três linhas de abate do frigorífico, uma é exclusiva para atender preceitos religiosos por meio do abate Halal, sem insensibilização e as outras duas atendem aos demais mercados consumidores e os animais são humanitariamente abatidos, empregando como insensibilização a eletronarcose, que possui parâmetros pré-definidos que são monitorados e verificados pelo estabelecimento na frequência preconizada no programa de autocontrole do elemento de inspeção de bem-estar animal.

\section{Diagnóstico de situação}

No mês de maio do ano de 2018 foi realizado um diagnóstico das condições do abatedouro em três dias aleatórios, no que concerne ao modo como este vinha sendo operado. De forma geral foram avaliadas as instalações, equipamentos e operações que envolvem o bem-estar no galpão de espera, dos animais alojados, plataforma de desembarque, pendura, insensibilização e sangria.

\section{Delineamento experimental}

Foram realizadas análises descritivas das informações coletadas, conforme ilustrações do fluxograma abaixo, o qual evidencia a localização e o diagnóstico realizado. 


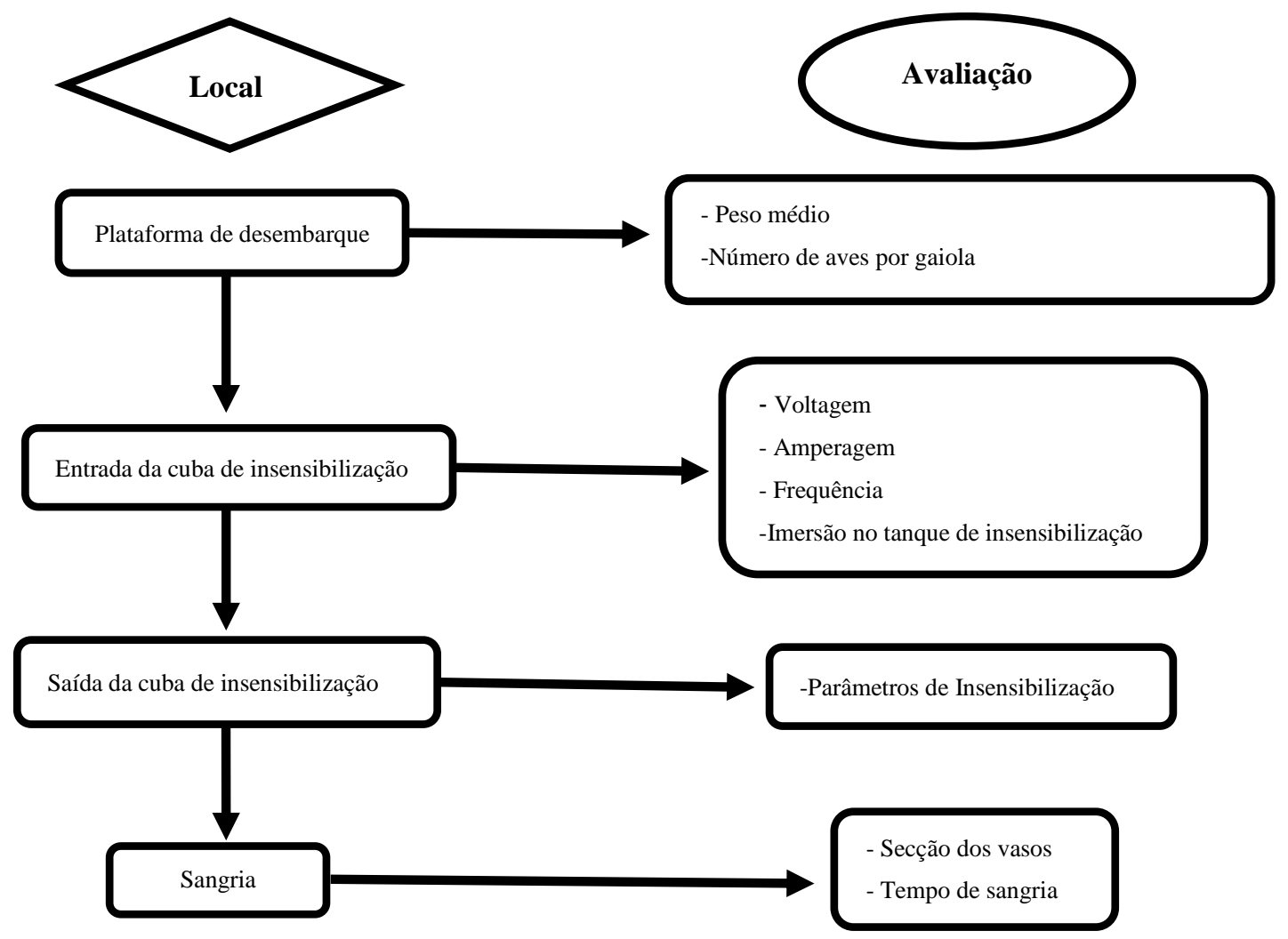

Fluxograma 1. Informações coletadas evidenciando a localização e o diagnóstico realizado no abatedouro-frigorífico da região sudoeste de Goiás.

\section{Resultados e discussão}

\section{Monitoramento do bem-estar durante o pré-abate e abate}

Manobras de bem-estar animal são adotadas em todas as etapas de produção dos frangos de corte, a fim de que se estabeleça o potencial máximo de produção dos animais alojados no galpão. Finalizado o processo de engorda a campo em granjas de produtores integrados à agroindústria, os frangos foram encaminhados para o abate em gaiolas carregadas de maneira sobreposta, em caminhões, com as laterais abertas e dispostas de modo que o ar circulasse entre as gaiolas, buscando-se evitar o estresse térmico das aves durante o transporte.

\section{Pré abate}

Finalizada a criação e engorda dos frangos a campo, estes são apanhados e dispostos em gaiolas, onde a lotação varia de acordo com o peso do lote, logo o conhecimento deste parâmetro é relevante, pois altas lotações de gaiolas podem comprometer o bem-estar das aves.

Chegando ao estabelecimento de abate os caminhões aguardam em um galpão de espera, o qual foi construído em meio às árvores nativas que sombreiam as adjacências do mesmo. O galpão possui 1.500 metros quadrados, totalmente abertos em pleno estado de manutenção e dotado com eficiente sistema de ventilação e aspersão em toda sua área comportando no máximo seis caminhões. O tempo médio de espera é variável e determinado pela demanda da indústria, porém o tempo máximo de jejum de 12 horas, que se inicia na granja, deve ser considerado a fim de evitar sofrimento aos animais. Nesse local a temperatura ambiente média de $27^{\circ} \mathrm{C}$, ventilação e umidade foram constantemente monitoradas, com média de $80 \%$ de umidade, mantendo tais parâmetros adequados às condições de bem-estar animal e evitando-se possível estresse térmico. De forma cadenciada, de acordo com a demanda do abate, os caminhões tinham acesso à plataforma de descarregamento.

Foram avaliados os caminhões utilizados no transporte das aves e a forma com que as caixas estavam dispostas. Os caminhões percorreram distancias médias de $60 \mathrm{~km}$, com uma média 5.500 animais. A média de animais mortos por caminhão foi de menos de $1 \%$ estando dentro da normalidade considerada. 


\section{Plataforma de desembarque}

Após a espera, as aves foram transportadas cuidadosamente até a área de recepção e desembarque dentro da fábrica. Assim como a área de espera, este local é protegido de condições climáticas adversas e fornece o suporte adequado para avaliação. A lotação de animais por gaiola foi avaliada, haja vista que uma quantidade inadequada de aves pode prejudicar o bem-estar das aves. A recepção das aves é feita em local arejado, com o intuito de proporcionar a ambiência adequada para evitar estresse térmico das aves. O descarregamento foi realizado de forma manual, colocando-se as caixas com os frangos direto em esteiras automáticas, tomando-se as devidas precauções a fim de evitar estresse desnecessário, como, por exemplo, minimizar ruídos, emissão de poeira e a elevação da temperatura ambiente com o intuito de manter ambiência adequada ao bem-estar das aves, evitar colisões entre gaiolas e mantendoas tampadas evitando-se fugas e aves soltas no pátio. Após essas observações avaliou-se o peso médio das aves e o número de aves por caixa, de acordo com programa de autocontrole de bem-estar animal verificado oficialmente pelo Serviço de Inspeção Federal. A quantidade de aves por gaiola identificada na linha foi de oito aves, com peso médio de $2,54 \mathrm{~kg} \pm 0,08 \mathrm{~kg}$ em 3.000 frangos avaliados.

\section{Pendura}

Da plataforma de recepção e descarga as caixas são encaminhadas pelas esteiras automatizadas para o setor de pendura onde os animais são retirados gentilmente das caixas e erguidos pelas pernas e colocados em ganchos dimensionados adequadamente para o tamanho das aves, considerando que o sistema de criação integrado permite a padronização do tamanho dos animais. A pendura foi realizada segurando as aves por ambas as pernas uma a uma, sem compressão excessiva das coxas. Durante esta operação de pendurar as aves pelos pés nos ganchos, pendurou-se uma ave por gancho, cada pé em seu respectivo lugar, ocupando-se todos os ganchos com o intuito de eliminar espaços vazios, de modo que o corpo de cada ave se apoiasse nos contíguos proporcionando possível sensação de imobilização que diminui a necessidade reativa da ave se debater.A área de pendura possuía iluminação diferenciada, por meio de luz negra e as nóreas possuíam parapeitos ao longo de toda a sua extensão até o início da cuba de insensibilização, a presença de parapeitos associado ao apoio contiguo das aves favorece a sensação de imobilização e diminui a necessidade de debate, observando-se, portanto o transporte calmo das aves até a cuba de insensibilização ou à sangria pelo método halal.Insensibilização

A avaliação dos parâmetros de eficiência da insensibilização foi obtida por meio da análise visual e por aferição do equipamento de choque com o uso de um instrumento calibrado em laboratório acreditado pela Rede brasileira de calibração RBC. Após o tanque de imersão das aves, com o auxílio de um contador numérico manual e de um cronometro digital, para cada parâmetro foram realizadas dez (10) repetições com cem (100) aves em cada repetição. Também podem ser observadas nesta tabela as médias das verificações para controle da voltagem $(119,6 \pm 1,19 \mathrm{~V})$, da amperagem $(3,49 \pm 0,08 \mathrm{~A}) \mathrm{e}$ da frequência $(399,86 \pm 0,35 \mathrm{~Hz})$, na cuba de insensibilização dos animais abatidos por eletronarcose seguido de sangria.

Tabela 1. Médias dos resultados, quando foram avaliados o peso, voltagem, amperagem e a frequência na cuba de insensibilização por eletronarcose, provenientes de um abatedouro-frigorífico de frangos situado no município de Rio Verde- GO, no ano de 2018

\begin{tabular}{l|ccc}
\hline Formas de abate & Voltagem, V & Amperagem, a & Frequência, Hz \\
\hline Frangos & $119,6 \pm 1,19$ & $3,49 \pm 0,08$ & $399,86 \pm 0,35$ \\
\hline
\end{tabular}

Decorrente da uniformidade constatada dos lotes experimentais, refletindo qualidade e padronização da criação a campo e sem grandes oscilações de peso vivo, verifica-se que os resultados da monitoração do processo de insensibilização atenderam ao Regulamento CE 1099/09 com reflexos positivos sobre os parâmetros de bem-estar.

A avicultura industrial busca a uniformização na criação dos animais, pois a mecanização é marcante nos frigoríficos e a regulagem das máquinas e equipamentos envolvidos na insensibilização depende da padronização do tamanho e peso dos animais. Os bons resultados apenas são possíveis com parceiros integrados, com histórico de bons controles nutricionais e sanitários, uniformidade de lotes, distância de percurso de transporte, duração do transporte e duração do pré-abate. Bressan \& Beraquet (2002) justificam que, quando ocorrem alterações na qualidade da carne, em animais que são do mesmo lote e 
que possuam mesma idade e sexo, é provável que essas decorram do estresse pré-abate, desencadeando transtorno fisiológico que pode causar alterações no processo de abate e na transformação do músculo em carne. Hildebrand et al. (2009) descrevem que a insensibilização incorreta ou parâmetros elétricos inadequadamente controlados no momento do choque podem ocasionar problemas nas carcaças, fatores que no momento da sangria podem ser evidenciados, justificando para tanto o controle da voltagem, amperagem e frequência do equipamento empregado na insensibilização. Conforme evidenciado anteriormente, verifica-se a adequação e correção do processo utilizado na amostragem experimental, uma vez que os parâmetros de choque encontravam-se dentro dos limites exigidos pela legislação, e atendendo portanto, aos procedimentos de bem-estar IN 03/2000 e o Regulamento CE 1099/09.

Em toda a extensão da área, da pendura até a entrada da cuba de insensibilização havia a instalação de artefato denominado parapeito, cujo objetivo também é o de amparar e acalmar as aves, diminuindo o grau de reatividade, além da existência de rampa inclinada imediatamente antes da entrada no tanque de insensibilização, cujo objetivo é o de evitar a ocorrência de pré-choques. Todo o conjunto mostrouse adequado às atividades, com bom estado de conservação e adequada manutenção. Nos setores de insensibilização e sangria a iluminação era reduzida 50 lux e, além disso, especificamente no setor de pendura a iluminação era realizada com luz negra, esta é o resultado da incidência da luz comum sobre o vidro de uma lâmpada especial, mais escura e sem fósforo. Nas lâmpadas fluorescentes brancas, a incidência da luz só é possível graças à camada de fósforo que reveste o tubo de vidro, isso porque a maior incidência é da luz ultravioleta, que só se torna visível quando passa pelo fósforo. Quando o fósforo é retirado e o vidro escurecido, a luz ultravioleta passa direta pelo vidro. Esta iluminação tem o intuito de acalmar as aves e diminuir o reflexo reacional de bater as asas.

Bem-estar é um termo utilizado para animais (Llonch et al., 2015). É considerado de importância especial por muitas pessoas; porém, requer uma definição estrita se a intenção é a sua utilização de modo efetivo e consistente. Um conceito claramente definido de bem-estar é necessário para utilização em medições científicas precisas, em documentos legais e em declarações e discussões públicas (Koknaroglu \& Akunal, 2013). Para que o bem-estar possa ser comparado em situações diversas ou avaliado em uma situação específica, deve ser medido de forma objetiva. Um critério essencial para a definição de bem-estar animal útil é que a mesma deve referir-se a característica do animal individual, e não a algo proporcionado ao animal pelo homem (Broom \& Molento, 2004). O bem-estar do animal pode melhorar como resultado de algo que lhe seja fornecido, mas o que se lhe oferece não é, em si, bem-estar. Os efeitos sobre o bem-estar incluem aqueles provenientes de doença, traumatismos, fome, estimulação benéfica, interações sociais, condições de alojamento, tratamento inadequado, manejo, transporte, procedimentos laboratoriais, mutilações variadas, tratamento veterinário (Broom \& Molento, 2004). Bem-estar deve ser definido de forma que permita pronta relação com outros conceitos, tais como: necessidades, liberdades, felicidade, adaptação, controle, capacidade de previsão, sentimentos, sofrimento, dor, ansiedade, medo, tédio, estresse e saúde (Carenzi \& Verga, 2009).

Se em dado momento um indivíduo não está em meio a adversidades, este indivíduo encontra-se provavelmente em homeostase completa, o que seria indicado na análise de parâmetros fisiológicos e de comportamento. Outro indivíduo pode passar por problemas, cuja magnitude ele não consiga enfrentar. Enfrentar com sucesso implica em ter controle da estabilidade mental e corporal. A dificuldade prolongada em se obter sucesso ao enfrentar uma dada situação, resulta em falência no crescimento, na reprodução e até em morte. Um terceiro indivíduo pode passar pelos problemas com dificuldade utilizando uma gama de mecanismos de adaptação. O segundo e o terceiro indivíduos têm alta probabilidade de demonstrar alguns sinais diretos de sua falência ou dificuldade na obtenção de sucesso ao enfrentar o problema. Muito provavelmente eles também experimentam sentimentos ruins associadas à sua situação. $\mathrm{O}$ bem-estar de um indivíduo é seu estado em relação às suas tentativas de adaptar-se ao seu ambiente (Broom, 1986). Esta definição refere-se a uma característica do indivíduo em um dado momento. A base do conceito é como o indivíduo está passando por uma determinada fase de sua vida, encontrando-se maiores detalhes nos relatos de Broom (1986), Broom (1991), Broom \& Johnson (1993) e Broom \& Molento (2004). O conceito refere-se ao estado de um indivíduo em uma escala variando de muito bom a muito ruim. Trata-se de um estado mensurável e qualquer avaliação deve ser independente de considerações éticas. 
Ao se considerar como avaliar o bem-estar de um indivíduo, é necessário haver de início um bom conhecimento da biologia do animal. O estado pode ser bom ou ruim; entretanto, em ambos os casos, além das mensurações diretas do estado do animal, devem ser feitas tentativas de se medir os sentimentos inerentes ao estado do indivíduo. Algumas medidas são mais relevantes aos problemas de curto-prazo, tais como aquelas associadas a manejo ou a um período breve de condições físicas adversas, enquanto outras são mais apropriadas a problemas de longo-prazo. Broom (1986), Fraser \& Broom (1997), Broom \& Johnson (1993) e Broom \& Molento (2004) proporcionam discussões detalhadas sobre medidas de bem-estar animal. Alguns sinais de bem-estar precário são evidenciados por mensurações fisiológicas. Por exemplo, aumento de frequência cardíaca, atividade adrenal, atividade adrenal após desafio com hormônio adrenocorticotrófico $(\mathrm{ACTH})$ ou resposta imunológica reduzida após um desafio podem indicar que o bem-estar está mais reduzido que em indivíduos que não mostrem tais alterações. Resultados das mensurações fisiológicas devem ser interpretados com cuidado, assim como aqueles de outras medidas descritas neste texto. O impedimento da função do sistema imune, assim como algumas outras alterações fisiológicas, pode indicar estado pré-patológico (Moberg, 1996). Mensurações do comportamento têm igualmente grande valor na avaliação do bem-estar. $\mathrm{O}$ fato de um animal evitar ou esquivar-se fortemente de um objeto ou evento fornece informações sobre seus sentimentos e, em consequência, sobre seu bem-estar. Quanto mais forte a reação de esquiva, mais pobre será o bem-estar durante a presença do objeto ou do fato. Um indivíduo que se encontra impossibilitado de adotar uma postura preferida de repouso, apesar de repetidas tentativas, será considerado como tendo um bem-estar mais pobre que outro cuja situação permite a adoção da postura preferida. Comportamentos anormais, tais como estereotipias, automutilação, bicar de penas em aves ou comportamento excessivamente agressivo indicam que o indivíduo em questão encontra-se em condições de baixo grau de bem-estar. Em algumas das avaliações fisiológicas e comportamentais citadas pode tornar-se óbvio que o indivíduo esteja tentando enfrentar situações adversas, e a extensão destas tentativas pode ser mensurada. Quaisquer que sejam as medições, os dados coletados em estudos de bem-estar animal fornecem informações sobre a posição do animal em uma escala de bem-estar, variando de um grau muito alto até muito baixo.

\section{Conclusão}

Considerando o diagnóstico de situação realizado, por seus resultados, se pode constatar o cumprimento dos critérios de bem-estar animal no manejo pré abate e plataforma de desembarque, que poderiam influenciar no abate, estando os mesmos de acordo com o direcionamento da (EFSA,2004), legislações europeias Regulamento (CE) n ${ }^{\circ} 099 / 2009$ e brasileiras (BRASIL, 2000) e (BRASIL, 2011).

\section{Referências bibliográficas}

BRASIL, 2000. Instrução Normativa ${ }^{\circ} 03$, de 17 de janeiro de 2000. Regulamento técnico de métodos de insensibilização para o abate humanitário animais de açougue. Secretaria de Defesa Agropecuária (DAS), Ministério da Agricultura, Pecuária e Abastecimento, Brasília.

BRASIL, 2011.Portaria n524, de 21 de junho de 2011. Institui a Comissão Técnica Permanente de Bem-Estar Animal - CTBEA, do Ministério da Agricultura, Pecuária e Abastecimento, com o objetivo de coordenar ações em bem-estar dos animais de produção e de interesse econômico nos diversos elos da cadeia pecuária. Ministerio da Agricultura, Pecuária e Abastecimento, Brasília.

BRASIL. Decreto $\mathrm{n}^{\circ} 9.667$, de 02 de janeiro de 2019. Aprova a Estrutura Regimental e o Quadro Demonstrativo dos Cargos em Comissão e das Funções de Confiança do Ministério da Agricultura, Pecuária e Abastecimento, remaneja cargos em comissão e funções de confiança, transforma cargos em comissão e funções de confiança e altera o Decreto ${ }^{\circ}$ 6.464, de 27 de maio de 2008, que dispõe sobre a designação e atuação de adidos agrícolas junto a missões diplomáticas brasileiras no exterior. Casa Civil, Presidência da República, Brasília, 2019. http://www.planalto.gov.br/CCIVIL 03/ Ato2019-2022/2019/Decreto/D9679.htm\#art10

Bressan, M. C. \& Beraquet, N. J. (2002). Efeito de fatores pré-abate sobre a qualidade da carne de peito de frango. Ciência e Agrotecnologia, 26(5):1049-1059.

Broom, D. M. (1986). Indicators of poor welfare. British Veterinary Journal, 142(6):524-526. 
Broom, D. M. (1991). Animal welfare: concepts and measurement. Journal of Animal Science, 69(10):4167-4175.

Broom, D. M. \& Johnson, K. G. (1993). Stress and animal welfare. London: Springer Science \& Business Media.

Broom, D. M. \& Molento, C. F. M. (2004). Animal welfare: concept and related issues-review. Archives of Veterinary Science, 9(2):1-11.

Carenzi, C. \& Verga, M. (2009). Animal welfare: review of the scientific concept and definition. Italian Journal of Animal Science, 8(1):21-30.

Conselho da União Europeia (CE). Regulamento (CE) n 1099/2009: Relativo à proteção de animais no momento da occisão. Jornal Oficial da União Europeia. (L303/1) 24 de setembro, 2009.

Dawkins, M. S. (2017). Animal welfare and efficient farming: is conflict inevitable? Animal Production Science, 57(2):201-208.

EFSA, 2004. Opinion of the Scientific Panel on Animal Health and Welfare on a request from the Commission related to welfare aspects of the main systems of stunning and Killing the main comercial species of animals. The EFSA jornal 45, 1-29.

Fraser, A. F. \& Broom, D. M. (1997). Farm animal behaviour and welfare. Cambridge: Cab International.

Grandin, T. (2014). Animal welfare and society concerns finding the missing link. Meat Science, 98461469.

Gregory, N. G. (2003). Animal welfare and the meat market. In N. G. Gregory (Ed.), Animal welfare and meat science (pp. 1-14). Cambridge: CABI.

Gregory, N. G. \& Grandin, T. (2007). Animal welfare and the meat market. Cambridge: CABI.

Hildebrand, C., Trowbridge, E., Roach, M. A., Sullivan, A. G., Broman, A. T. \& Vogelman, B. (2009). Resident self-assessment and self-reflection: University of Wisconsin-Madison's five-year study. Journal of General Internal Medicine, 24(3):361-365.

Koknaroglu, H. \& Akunal, T. (2013). Animal welfare: An animal science approach. Meat Science, 95(4):821-827.

Llonch, P., King, E. M., Clarke, K. A., Downes, J. M. \& Green, L. E. (2015). A systematic review of animal based indicators of sheep welfare on farm, at market and during transport, and qualitative appraisal of their validity and feasibility for use in UK abattoirs. The Veterinary Journal, 206(3):289297.

Moberg, G. P. (1996). Suffering from stress: an approach evaluating the welfare of an animal. Acta Agriculturae Scandinavica, Section A46-49.

Recebido: 2 de abril, 2019.

Aprovado: 15 de maio, 2019.

Publicado: 21 de maio, 2019.

Licenciamento: Este artigo é publicado na modalidade Acesso Aberto sob a licença Creative Commons Atribuição 4.0 (CC-BY 4.0), a qual permite uso irrestrito, distribuição, reprodução em qualquer meio, desde que o autor e a fonte sejam devidamente creditados. 